\title{
Radial-maze learning by lines of taste-aversion-prone and taste-aversion-resistant rats
}

\author{
STEPHEN H. HOBBS \\ Augusta College, Augusta, Georgia
}

and

\author{
PAUL A. WALTERS III, ELIZABETH F. SHEALY, and RALPH L. ELKINS \\ Veterans Affairs Medical Center and Medical College of Georgia, Augusta, Georgia
}

\begin{abstract}
Lines of taste-aversion-prone and taste-aversion-resistant rats have been developed through 22 generations of bidirectional selective breeding for efficient or inefficient taste-aversion conditionability. The present study compared these two lines on food-reinforced foraging in a radialarm maze. The lines, although not identical on all measures taken, were equally adept at learning the maze. This finding supports prior indications that selective breeding has exerted an effect on learning that is highly specific to taste-aversion conditionability. Therefore, the genetically based line differences in taste-aversion conditionability cannot be attributed to a genetic difference in general learning aptitude.
\end{abstract}

Nausea-based conditioned taste aversions (CTAs) are acquired efficiently by rats and humans (Garcia, 1989). Nevertheless, considerable individual variability in CTA is observed within each species. For humans, individual differences in taste-aversion (TA) conditionability may modulate the effectiveness of nausea-based consummatory aversion treatments of alcohol and drug dependence (Elkins, 1980, 1991). Additionally, individual differences in CTA propensities may influence cancer patients' development of aversions to foods consumed in association with chemotherapy-induced nausea (Bernstein, 1978).

The rat has been advanced as a useful TA learning model of nausea-based consummatory aversion therapies (Elkins, 1980; Logue, 1985). In an attempt to clarify possible genetic contributions to individual differences in TA learning propensities, Elkins (1986) undertook a program of CTA-based bidirectional selective breeding of SpragueDawley-derived rats. Selection was initiated at each of the two extremes of TA conditionability in randomly bred stock. At approximately 90 days of age, nonsibling matings were effected between the best learners and between the poorest learners of a cyclophosphamide-induced CTA to a normally preferred saccharin solution.

An overview of the CTA methodology, which is detailed elsewhere (Elkins, 1986), is as follows: Fluiddeprived rats ingest a normally palatable saccharin solution and then are injected with cyclophosphamide. Cy-

This research was supported by the VA Medical Research Service and by NIAAA Grant RO-1 AA06465. The authors wish to thank J. Martin, F. Westbrook, E. Hudson, A. Brandsma, G. Baker, and K. Shealy for their assistance with this study. Correspondence should be addressed to R. L. Elkins, Psychology Research 116 BU, V.A. Medical Center, Augusta, GA 30910. clophosphamide produces nausea in humans (Bernstein, 1978) and, presumably, elicits some comparable experience within rats, as indicated by its effectiveness as a TA conditioning agent. After a 3-day recovery period from the acute effects of cyclophosphamide, the rats begin 15 days of preference testing. During this assessment, they have continuous access to the saccharin solution and to plain tap water. A daily preference score is computed that indicates the percentage to which ingestion of the saccharin solution contributes to total daily fluid intake. A subject's mean saccharin preference score, averaged over an interval of at least 3 days, has been the behavioral index of breeder selection.

Line differences in TA conditionability appeared within the 2nd selected (S-2) generation (Elkins, 1986). Ongoing selection across 22 additional generations has produced TA-prone (TAP) and TA-resistant (TAR) rats that now display marked between-line separation and low within-line variabilities in CTA acquisition. For example, the 22nd selected (S-22) TAP and TAR generations' mean saccharin preference scores during 3 days of postconditioning preference testing were $5.24 \pm 0.30(M+S E)$ and $94.64 \pm 5.24$, respectively.

The following standard control procedures support a conditioning (associative) interpretation of the TAP and TAR preference-score difference. It has been repeatedly demonstrated that the TAP rats do not form TAs to the saccharin solution (1) if they are injected with normal saline instead of cyclophosphamide after their initial saccharin intake or (2) if they drink plain tap water prior to cyclophosphamide administration and, thereafter, encounter the saccharin solution as a novel flavor at the onset of two-bottle preference testing. It has also been demonstrated that the line differences in CTA learning are not 
dependent on the saccharin solution or on the postinjectional consequences of cyclophosphamide that served as the respective conditioned stimulus (CS) and unconditioned stimulus (US) during line selection. Characteristic line differences in CTA acquisition have been demonstrated with alternate CS flavors (beef- and maple-flavored solutions; Elkins, 1983) and within a cricket-predation TA paradigm (Elkins, Gerardot, \& Hobbs, 1989). Lineseparating US alternatives to cyclophosphamide included rotational stimulation (i.e., motion sickness; Elkins, Walters, Harrison, \& Albrecht, 1990) and an intraperitoneal injection of lithium chloride, emetine hydrochloride, ethanol (Elkins, Walters, \& Orr, 1992), or cocaine hydrochloride (Elkins et al., 1991).

The TAP and TAR phenotypes have been developed, in part, to serve as subjects in studies of the biological mechanisms that subserve TA acquisition, maintenance, and extinction (Aronstam, Elkins, \& Walters, 1990; Orr, Walters, Carl, \& Elkins, in press). A major concern in interpreting the results of such experimentation, however, is whether the TAP and TAR differences in conditionability are specific to the TA paradigm or are manifestations of more encompassing selectively bred line differences in general learning capacities.

Prior to the beginning of line development, it was hypothesized that selective breeding based solely on TA behavioral phenotypes would produce TAP and TAR rats that remained indistinguishable with respect to shockmotivated environmental avoidance (SMEA) learning (i.e., the learning of a passive-avoidance response to a shuttlebox compartment that previously had been paired with escapable shock; Elkins, 1986). This prediction was based (1) on the Garcia and Ervin (1968) neuroanatomical diversity hypothesis, which holds that illness-induced TA and SMEA responses are subserved by neuroanatomically discrete associative mechanisms, and (2) on the results of brain-lesion experiments that selectively disrupted either TA or SMEA learning (Elkins, Fraser, \& Hobbs, 1977; Elkins, Jerald, \& Hobbs, 1975; McGowan, Garcia, Ervin, \& Schwartz, 1969; McGowan, Hankins, \& Garcia, 1972). Seligman's (1970) concept of associative preparedness and Rozin and Kalat's (1971) position that the laws governing TAs are not necessarily identical to those that govern other types of avoidance learning were also judged to be consistent with the possibility of breeding for differences in TA without altering SMEA conditioning capabilities.

As hypothesized, the selective breeding separated the CTA propensities of the two lines with no corresponding line separation of SMEA learning (Elkins, 1986). TAP and TAR rats have also been found to be equivalent learners of barpress responding under both fixed-ratio and differential-reinforcement-of-low-rate schedules of food reinforcement (Hobbs \& Elkins, 1983). The present study compared TAP and TAR rats with respect to another learning task, the food-reinforced acquisition of efficient foraging in a radial-arm maze. As an appetitively based spatial problem, the radial-arm maze is distinct from previously employed tasks. Furthermore, it represents a laboratory analog to natural foraging used by rodents $(\mathrm{Ol}-$ ton, 1982).

\section{METHOD}

\section{Subjects}

The subjects were 12 TAP and 12 TAR rats, including equal numbers of males and females in each line, from the S-22 generations. Mean age at the beginning of the experiment was 263 days (range 252-265). The rats were bred in the Animal Research Facility at the Veterans Affairs Medical Center, Augusta, Georgia. The colony room was maintained on a 12:12-h light:dark cycle, with lights off at noon EST. Ambient temperature was held at $23 \pm 2^{\circ} \mathrm{C}$. The rats were caged individually and, except as described below, had free access to water and rat chow prior to the study. Routine maintenance was carried out in the last hour of light, and testing took place in an adjacent room during the first $3 \mathrm{~h}$ of the dark period.

\section{Apparatus}

An eight-arm radial maze was constructed out of plywood, painted with black enamel, and coated with polyurethane. Arms were $88.9 \mathrm{~cm}$ in length and $15.2 \mathrm{~cm}$ wide, with walls $16.5 \mathrm{~cm}$ high. The arms radiated from an octagonal centerpiece measuring $36.8 \mathrm{~cm}$ across facing sides. The apparatus was $84 \mathrm{~cm}$ above the floor. A small, black plastic cup was fastened to the floor at the end of each maze arm to serve as a holder for food reinforcers. Overhead illumination sufficient for observation was provided by a red incandescent bulb.

\section{Procedure}

The animals were reduced to $80 \% \pm 2 \%$ of their free-feeding weights by reducing the amount of available food. These target weights were maintained on the first 5 days of testing, with increases to $85 \% \pm 2 \%$ of the free-feeding weights permitted on the second 5 days of testing.

Three squads of 8 rats each, matched for sex and line, were randomly assigned to groups with two observer-handlers in charge of each squad. Each rat's line designation was unknown to the observer-handlers throughout the behavioral testing. The subjects were trained in the same order each day for 10 consecutive days, weekend excluded.

Prior to each trial, the maze was washed and all arms were baited with a food kernel (one third of a piece of Kellogg's Fruit Loops cereal). The animals were placed alone in the center compartment, always facing the same direction. After being released, the subjects were permitted to move about the maze without restriction until all cereal kernels had been consumed or $5 \mathrm{~min}$ had elapsed, whichever occurred first. The observers recorded the number of arms entered, the number of arms reentered, the number of times a food well was reached, the number of kernels consumed, the number of reentry errors (reentering an arm where the kernel had been consumed), the number of rearings, and any instances of urination or defecation. Arm entry or reentry was defined as a subject's placement of its forepaws within a maze arm.

\section{RESULTS}

Each dependent measure was analyzed with a $2 \times 2 \times 10$ (line $\times \operatorname{sex} \times$ test day) mixed analysis of variance, with test day serving as the repeated measure. To maintain sufficient power to detect potential differences between the two lines, no correction was made for the number of mixed analyses performed.

No line differences were found with respect to maze learning. The number of reentry errors changed over the 10 test days $[F(9,180)=6.64, p<.001]$; however, there was no line effect $[F(1,20)=1.49, p=.24]$ or line $\times$ day interaction $[F(9,180)=.72, p=.69]$ (see Figure 1A). Similarly, there was a decrease across days of 
testing in the foraging time that was required to consume all eight rewards $[F(9,180)=20.38, p<.001]$ (see Figure 1B).

There were no statistically significant differences between the TAP and TAR rats $[F(1,20)=3.62, p=.071]$.

No sex differences materialized on any measure. However, a significant line $\times$ sex interaction on number of food kernels consumed was obtained $[F(1,20)=4.35$, $p=.05]$; female TAR rats ate more kernels than did female TAP rats $[6.9 \pm 0.3(M \pm S E)$ and $4.8 \pm 1.0$, respectively].

The TAP rats reared more than did the TAR rats $[F(1,20)=8.53, p=.008$, TAP $=15.3 \pm 1.4$, TAR $=$ 11.6土1.5], with rearing decreasing across training for both lines $[F(9,180)=15.61, p<.001]$ (see Figure 2A). No main effects for line were found with respect to the total number of arms entered (the number of arms entered plus the number of arms reentered), the number of arms reentered, or the number of food reservoirs reached $(p s>.15)$. Significant line $\times$ day interactions $[F \mathrm{~s}(9,180)$ $\geq 2.31, p s \leq .017]$ and subsequent individual comparisons confirmed that the TAP rats were more active in terms of moving about in the maze only during the first 3 days of testing (see Figure 2B).

\section{DISCUSSION}

The principal measures of radial-arm-maze task acquisition (reentry errors and time to complete foraging) revealed no line differences. However, the TAP rats were found to be somewhat more active in the maze than were the TAR rats, especially during the first 3 days of testing.

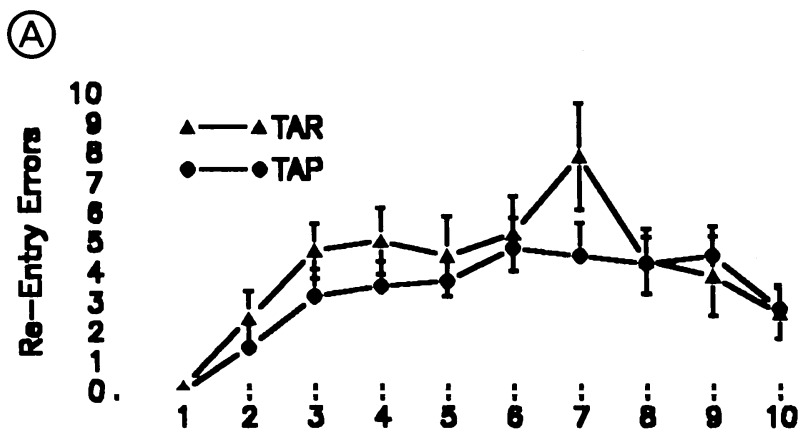

(B)

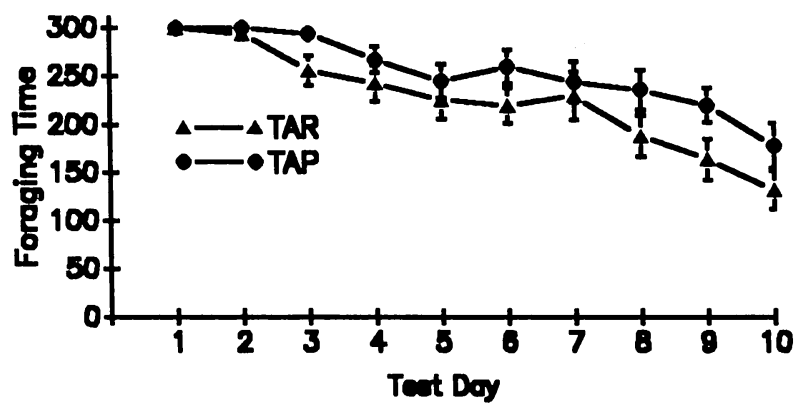

Figure 1. Measures of performance. (A) $\mathrm{Me}^{\mathrm{e}_{a n}}$ number of reentry errors across test days. (B) Mean time (in seconds) to consume all reinforcers across test days.

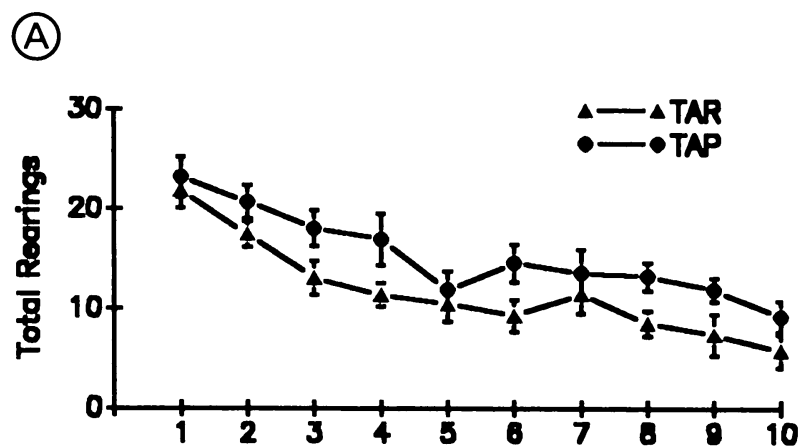

(B)

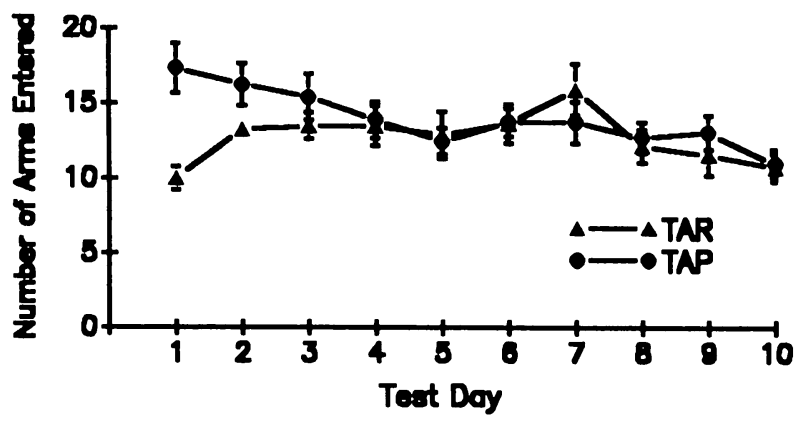

Figure 2. Measures of activity in the radial-arm maze. (A) Mean number of rearings while in the radial-arm maze across test days. (B) Mean number of arms entered (defined as the number of arms entered plus the number of arms reentered) while in the radial-arm maze across test days.

The results of this experiment are consistent with previous studies conducted on earlier generations of TAP and TAR rats that have shown little or no evidence that the two lines perform differently on learning tasks other than TA conditioning (Elkins, 1986; Hobbs \& Elkins, 1983). Such a partitioning of TA and non-TA learning is consistent with the results of brain-lesion experiments that have demonstrated neuroanatomically distinguishable systems subserving TA acquisition and other types of learning (McGowan et al., 1969; McGowan et al., 1972; Rozin \& Kalat, 1971; Seligman, 1970).

The possibility remains that TAP and TAR differences in maze learning might have emerged if trials had been continued to an errorless criterion. Although this seems unlikely given the trends in the present data, replication of the present experiment with subsequent TAP and TAR generations will use a mastery criterion.

It appears that the breeding program has exerted a strong selective effect on the gene(s) that mediate TA learning without coincident selection of gene(s) that subserve other varieties of learning. This conclusion implies that the two inbred lines are indistinguishable on the basis of a generalized learning ability. The possibility remains, however, that the line differences detected in the present study with respect to activity and rearings could differentially affect performance on other types of learning. These findings merit scrutiny in future investigations. Nevertheless, the use of TAP and TAR rats, given their marked between-line CTA separation and minimal within-line CTA variabilities, should enhance the statistical power of studies that examine the biological and neuroanatomical bases of CTA phenomena. The findings of such studies should clarify basic CTA processes. They also might have application to improved consummatory aversion treatments of human alcoholism and other drug dependencies.

\section{REFERENCES}

Aronstam, R. S., Elkins, R. L., \& Walters, P. A. (1990). Muscarinic acetylcholine receptor densities in brains of genetically selected 
taste-aversion-prone and resistant rats. Medical Science Research, 18, 213-214.

BERNSTEIN, I. L. (1978). Learned taste aversions in children receiving chemotherapy. Science, 200, 1302-1303.

ElkINS, R. L. (1980). Covert sensitization treatment of alcoholism: Contributions of successful conditioning to subsequent abstinence maintenance. Addictive Behaviors, 5, 67-89.

ELkINS, R. L. (1983). [CS substitution: Beef- and maple-flavored solutions]. Unpublished raw data.

ElKiNs, R. L. (1986). Separation of taste aversion prone and resistant rats through selective breeding: Implications for individual differences in conditionability and aversion therapy alcoholism treatment. Behavioral Neuroscience, 100, 121-124.

ElKINS, R. L. (1991). An appraisal of chemical aversion (emetic therapy) alcoholism treatment. Behaviour Research \& Therapy, 29, 387-413.

Elkins, R. L., Fraser, J., \& Hobbs, S. H. (1977). Differential olfactory bulb contributions to baitshyness and place avoidance learning. Physiology \& Behavior, 19, 787-793.

Elkins, R. L., Gerardot, R. J., \& HobBs, S. H. (1989). Differences in cyclophosphamide induced suppression of cricket predation in selectively-bred strains of taste-aversion prone and resistant rats. Behavioral Neuroscience, 103, 112-116.

Elkins, R. L., JeRALD, J. L., \& HobBs, S. H. (1975). Dissociation of shock-motivated passive avoidance and illness-induced taste aversions by habenula lesions. Neuroscience Abstracts, 1, 478.

Elkins, R. L., Walters, P. A., Harrison, W. R., \& Albrecht, W. (1990). Congruity of rotational and pharmacological taste aversion (TA) conditioning within strains of selectively bred TA prone and TA resistant rats. Learning \& Motivation, 21, 190-198.

Elkins, R. L., Walters, P. A., \& OrR, T. E. (1992). Continued development and unconditioned stimulus characterization of selectivelybred lines of taste aversion prone and resistant rats. Alcoholism: Clinical \& Experimental Research, 16, 928-934.

Elkins, R. L., Walters, P. A., Orr, T. E., Kolbe, E. F., West-
BROOK, F., \& НоввS, S. Н. (1991). Taste aversion inducing effects of cocaine in selectively-bred taste aversion prone and resistant rats. Neuroscience Abstracts, 17, 662.

GarCIA, J. (1989). Food for Tolman: Cognition and cathexis in concert. In T. Archer \& L. Nilsson (Eds.), Aversion, avoidance, and anxiety: Perspectives on aversively motivated behavior (pp. 45-85). Hillsdale, NJ: Erlbaum.

GaRCIA, J., \& Ervin, F. R. (1968). Gustatory-visceral and telereceptorcutaneous conditioning-adaptation in internal and external milieus. Community Behavioral Biology, 1(Pt. A), 389-415.

Hoвbs, S. H., \& ElKins, R. L. (1983). Operant performance of rats selectively bred for strong or weak acquisition of conditioned taste aversions. Bulletin of the Psychonomic Society, 21, 303-306.

Logue, A. W. (1985). Conditioned food aversion learning in humans. Annals of the New York Academy of Science, 443, 316-329.

McGowan, B. K., Garcia, J., ERvin, F. R., \& Schwartz, J. (1969). Effects of septal lesions on bait-shyness in the rat. Physiology \& Behavior, 4, 907-909.

McGowan, B. K., Hankins, W. G., \& Garcia, J. (1972). Limbic lesions and control of the internal and external environment. Journal of Behavioral Biology, 7, 841-852.

Olton, D. S. (1982). Spatially organized behavior in animals: Behavioral and neurological studies. In M. Potgral (Ed.), Spatial abilities: Developmental and physiological foundations (pp. 335-360). New York: Academic Press.

Orr, T. E., Walters, P. A., Carl, G. F., \& Elkins, R. L. (in press). Taste aversion resistant rats have higher NE and lower 5-HT levels in brain than taste aversion prone rats. Physiology \& Behavior.

Rozin, P., \& Kalat, J. (1971). Specific hungers and poison avoidance as adaptive specialization of learning. Psychological Review, 78 459-486.

Seligman, M. E. P. (1970). On the generality of the laws of learning. Psychological Review, 77, 406-418.

(Manuscript received October 1, 1992.) 